\title{
Tecendo redes: itinerários terapêuticos de pessoas com estomia
}

\author{
Weaving networks: therapeutic itineraries of people with ostomy \\ Redes de tejido: itinerarios terapéuticos de personas con ostomia
}

Antonio Dean Barbosa Marques ${ }^{1 *}$, Ezequias Alexandre da Silva², Samara Aurélio Sales Vidal2, Thereza Maria Magalhães Moreira', Virna Ribeiro Feitosa Cestari', Raquel Sampaio Florêncio ${ }^{1}$

\section{ORCID IDS}

Marques ADB (D) https://orcid.org/0000-0001-8969-1546

Silva EA (D) https://orcid.org/0000-0002-9075-8514

Vidal SAS (D) https://orcid.org/0000-0001-8321-9320

Moreira TMM (D) https://orcid.org/0000-0003-1424-0649

Cestari VRF (D) https://orcid.org/0000-0002-7955-0894

Florêncio RS (D) https://orcid.org/0000-0003-3119-7187

\section{COMO CITAR}

Marques ADB; Silva EA; Vidal SAS; Moreira TMM; Cestari VRF; Florêncio RS. Tecendo redes: itinerários terapêuticos de pessoas com estomia. ESTIMA, Braz. J. Enterostomal Ther., 2020, 18: e2120. https://doi.org/10.30886/estima.v18.909_PT

\section{RESUMO}

Objetivo: descrever o itinerário terapêutico de pessoas com estomia em busca de cuidados especializados. Método: estudo exploratório, descritivo, com abordagem qualitativa, com dez pessoas com estomia atendidas no Serviço de Atenção à Saúde da Pessoa Ostomizada, de agosto a novembro de 2017. A reconstituição se deu por meio de entrevistas semiestruturadas, registro em diário de campo e consulta aos prontuários dos serviços de saúde especializados, e processadas pelo software Interface de $R$ pour les Analyses Multidimensionnelles de Texte et de Questionnaires. Resultados: o itinerário terapêutico tem início com a descoberta dos sinais e sintomas, quando há acesso aos serviços de saúde mais próximos e a posteriori em busca de serviço especializado. Marcado por conexões formais e sustentados por subsistemas inter-relacionados. As trajetórias de busca, produção, transporte sanitário e gerenciamento do cuidado para saúde, empreendidos por pessoas, famílias e grupos informais expressam percursos e movimentos distintos na fase que antecede ao atendimento especializado. Conclusão: o conhecimento apreendido pelos discursos possibilita a identificação de necessidades básicas apresentadas por essa clientela, facilidades e barreiras encontradas no percurso empreendido para obter um plano terapêutico adequado.

DESCRITORES: Estomaterapia; Estomia; Aceitação pelo paciente de cuidados de saúde; Acesso aos serviços de saúde; Atenção à saúde.

\footnotetext{
1. Universidade Estadual do Ceará - Departamento de Enfermagem - Programa de Pós-Graduação em Cuidados Clínicos em Enfermagem e Saúde - Fortaleza (CE), Brasil.

2. Faculdade Princesa do Oeste - Departamento de Enfermagem - Curso de Graduação em Enfermagem - Crateús (CE), Brasil.

*Autor correspondente: antonio-dean@hotmail.com

Recebido: Jun. 26, 2020 | Aceito: Ago. 26, 2020
} 


\section{ABSTRACT}

Objective: To describe the therapeutic itinerary of people with an ostomy in search of specialized care. Method: exploratory, descriptive study with a qualitative approach, with ten people with ostomy treated at the Ostomy Care Service (Serviço de Atenção à Saúde da Pessoa Ostomizada), from August to November 2017. Reconstitution took place through semi-structured interviews, registration in a field diary and consultation with records of specialized health services, and processed by the software Interface de $R$ pour les Analyses Multidimensionnelles de Texte et de Questionnaires. Results: the therapeutic itinerary begins with the discovery of signs and symptoms, when there is access to the nearest health services and later in search of specialized services. Marked by formal connections and supported by interrelated subsystems. The trajectories of search, production, health transport and health care management undertaken by individuals, families and informal groups express different paths and movements in the phase that precedes specialized care. Conclusion: the knowledge apprehended by the speeches enables the identification of basic needs presented by this clientele, facilities and barriers encountered in the course undertaken to obtain an adequate therapeutic plan.

DESCRIPTORS: Stomatherapy; Stoma; Patient acceptance of health care; Access to health services; Health care.

\section{RESUMEN}

Objetivo: Describir el itinerario terapéutico de las personas con ostomía en busca de atención especializada. Método: Estudio exploratorio descriptivo, con un enfoque cualitativo, con diez personas con ostomía tratadas en el Servicio de Cuidado de Ostomía, de agosto a noviembre de 2017. La reconstitución se realizó mediante entrevistas semiestructuradas, registro en un diario de campo y consulta. A los registros de servicios de salud especializados, procesados por el software Interface de $R$ pour les Analyzes Multidimensionnelles de Texteset et de Questionnaires. Resultados: El itinerario terapéutico comienza con el descubrimiento de signos y síntomas, cuando hay acceso a los servicios de salud más cercanos y a posteriori en busca de servicios especializados. Marcado por conexiones formales, respaldado por subsistemas interrelacionados. Las trayectorias de búsqueda, producción, transporte de salud y gestión de la atención de salud, emprendidas por personas, familias y grupos informales, expresan diferentes caminos y movimientos en la fase que precede a la atención especializada. Conclusión: El conocimiento aprehendido por los discursos permite identificar las necesidades básicas presentadas por esta clientela, las instalaciones y las barreras encontradas en el curso realizado, a fin de obtener un plan terapéutico adecuado.

DESCRIPTORES: Estomaterapia; Estoma; Aceptación del paciente de atención médica; Acceso a servicios de salud; Cuidado de la salud.

\section{INTRODUÇÃO}

A prestação de atenção aos usuários de serviços de saúde deve primar pela integralidade do cuidado. Essa se torna factível quando a atenção se dá em rede. Logo, cada serviço deve ser repensado como elemento imprescindível, uma estação no circuito que cada pessoa percorre para obter a integralidade de que necessita ${ }^{1}$.

No entanto, os sistemas fragmentados são predominantes, separados e sem interlocução entre os serviços, como a atenção primária com a secundária ou terciária. Por sua vez, os sistemas integrados constituem a Rede de Atenção à Saúde e primam por adjuntos coordenados de unidades para proporcionar assistência constante e integral à população estabelecida ${ }^{2}$. Embora conjecture-se a relevância dos mecanismos de referência e contrarreferência, pondera-se suas pactuações, de modo a reordenar os processos de trabalho perante Linhas de Cuidado Integral em Saúde 3 . Desse modo, proporcionar o acesso a todos os recursos tecnológicos que o usuário necessita, integrando ações preventivas, curativas e de reabilitação, efetivadas por fluxos assistenciais como se fosse uma representação gráfica do itinerário dentro da rede 4 .

Em busca de romper com a fragmentação e descontinuidade do cuidado e da gestão nas regiões de saúde e com vistas a aperfeiçoar o funcionamento políticoinstitucional do Sistema Único de Saúde para assegurar ao usuário o conjunto de ações e serviços de que necessita com efetividade e eficiência, criou-se como estratégia a Rede de Atenção à Saúde por meio da Portaria no 4.279, de 30 de dezembro de $2010^{5}$.

Destaca-se dentre as redes, a Rede de Cuidados à Pessoa com Deficiência, que articula pontos de atenção à saúde para pessoas com deficiência temporária ou permanente, nas formas progressiva, regressiva ou estável, sendo intermitente ou contínua, no âmbito do Sistema Único de Saúde, a fim de iniciar precocemente ações de reabilitação e prevenção de incapacidades ${ }^{6}$.

Dentre as pessoas com deficiência física, destaca-se o indivíduo com estomia. A historicidade das Políticas Públicas em Saúde na atenção à saúde da pessoa com estomia tem passado por diversas transformações ao longo do tempo no 
mundo e no Brasil. Entre os marcos legais, destacam-se, em 2009, a publicação da portaria no 400 , na qual foram estabelecidas as Diretrizes Nacionais para a Atenção à Saúde das Pessoas Ostomizadas no âmbito do Sistema Único de Saúde e a criação do Serviço de Atenção à Saúde da Pessoa Ostomizada ${ }^{7}$ e a resolução normativa no 325 no âmbito de saúde suplementar ${ }^{8}$.

Ressalta-se que apesar do termo ostomia não existir na língua portuguesa, esse se mantém quando se trata de políticas públicas. Neste estudo adotamos o termo estomia.

O Serviço de Atenção à Saúde da Pessoa Ostomizada tem como objetivo realizar ações para promoção do autocuidado, prevenção e tratamento de complicações nas estomias, fornecimento de equipamentos coletores e adjuvantes de proteção e segurança e capacitação de profissionais ${ }^{7,9}$.

Coadunado às diretrizes nacionais estabelecidas pelo Ministério da Saúde, no estado do Ceará foi implementado em 2016 por meio da Secretaria da Saúde, localizada em Fortaleza, Ceará, Brasil. Esse capacita pessoas com estomia para o autocuidado, tratamento e reabilitação, distribui equipamentos coletores e dispositivos adjuvantes, além de prestar atendimento de equipe multiprofissional e contar com fluxo para reversão de estomia ${ }^{10}$.

$\mathrm{O}$ aumento expressivo de pessoas com estomias no estado, com média mensal de 60 casos novos ${ }^{10}$, e a extensão territorial do estado, com municípios distantes da capital, torna essencial o conhecimento do percurso desses pacientes em busca de cuidados. Esse conhecimento pode proporcionar atividades de promoção da saúde mais singulares e inclusivas. Os estudos dos itinerários terapêuticos proporcionam identificar o trajeto percorrido - no que se refere ao fluxo formal. Além de revelar as escolhas, o acesso ao atendimento especializado e à rede social na qual a pessoa faz parte.

O estudo torna-se relevante por compor a Agenda Nacional de Prioridades de Pesquisa em Saúde no que tange ao eixo: avaliação de políticas, programas e serviços; subitem: acessibilidade aos serviços de saúde ${ }^{11}$. Diante do exposto, emergiu a seguinte questão norteadora: quais os caminhos percorridos pela pessoa com estomia até chegar ao serviço especializado?

\section{OBJETIVO}

Descrever o itinerário terapêutico de pessoas com estomia em busca de cuidados especializados.

\section{MÉTODOS}

Estudo exploratório, descritivo, com abordagem qualitativa. O estudo foi realizado no município de Crateús, Ceará, Brasil, de agosto a novembro de 2017.

Foram identificadas 23 pessoas adultas cadastradas no Programa pertencente ao município de Crateús. Devido à ausência de números de telefones no cadastro de alguns pacientes, 13 foram contatados via telefone e aceitaram participar do estudo após esclarecimento do objetivo e método do estudo. Durante o contato presencial três pessoas solicitaram desistência, alegando não possuir disponibilidade de tempo para realizar a(s) entrevista(s).

Assim, participaram do estudo dez pessoas com estomia, residentes em Crateús e em acompanhamento pelo Programa de Atenção à Saúde da Pessoa Ostomizada do Ceará. O tamanho da amostra foi delimitado por ocorrência discursiva dos depoimentos ${ }^{12}$. Adotou-se como critérios de inclusão: idade a partir de 18 anos, residir em Crateús, ter estomia intestinal ou urinária e ser acompanhado pelo programa. Foram excluídas pessoas com comprometimento cognitivo que limitasse a participação na pesquisa por não compreenderem a entrevista.

Para coleta de dados, utilizaram-se dois instrumentos. $\mathrm{O}$ primeiro foi um questionário com perguntas fechadas sobre variáveis sociodemográficas e clínicas (sexo, idade, estado civil, tipo de estomia, tempo de convívio com a estomia, etiologia da estomia e renda pessoal). O segundo instrumento foi um roteiro de entrevista semiestruturada, fundamentado na literatura acerca da temática e experiência profissional com o público-alvo, composto de questões de identificação dos participantes e subitens na forma de questões disparadoras, sobre a vida antes e após a construção da estomia e a(s) "linha(s) de produção de cuidado" (continuum assistencial).

Os participantes foram abordados via ligação telefônica e foi agendado dia, horário e local de sua preferência para a aplicação da entrevista, conforme a disponibilidade. As entrevistas foram gravadas em aparelho smartphone - modo offine, contendo aplicativo de gravador digital.

Foram realizadas entrevistas em profundidade com os participantes, anotações em diário de campo e consulta aos prontuários dos serviços de saúde, conformando o material empírico central a partir do qual foram construídas dez narrativas, que expressam a experiência da busca por cuidado.

Ademais, foi empregado diário de campo para registro de aspectos relevantes observados durante a entrevista, 
objetivando retratar os sujeitos e reconstruir o diálogo. Com objetivo de garantir o anonimato aos participantes cujos itinerários terapêuticos foram analisados, adotou-se a letra "D”, de depoente, seguida de um algarismo arábico na sequência da realização das entrevistas.

Ressalta-se que para alguns participantes foram realizadas duas a três entrevistas presenciais, além dos contatos telefônicos e conversas com familiares na tentativa de complementar as informações necessárias. Em caráter complementar, para ajudar a compor o itinerário terapêutico, foi realizada também consulta ao prontuário do paciente, efetuada a partir da identificação dos serviços por onde o usuário passou até acessar o serviço especializado.

As entrevistas foram transcritas na íntegra para o programa LibreOffice, submetidos os dados a refinamento para exclusão de repetições vocabulares, agrupamento das palavras por aproximação semântica e composição de dicionário. Elaborou-se o corpus e, em seguida, realizou-se a análise de similitude com o processamento pelo software Interface de $R$ pour les Analyses Multidimensionnelles de Texte et de Questionnaires (IRaMuTeQ). Trata-se de um programa gratuito, que se ancora no software $\mathrm{R}$ e favorece diferentes formas de análises estatísticas em relação ao corpus textuais e sobre tabelas de indivíduos por palavras $^{13}$. Ressalta-se que os dados foram contrastados à luz da literatura sobre a temática.

O estudo obedeceu a Resolução 466/2012 do Conselho Nacional de Saúde e foi submetido, analisado e aprovado pelo Comitê de Ética em Pesquisa, sob parecer número 2.254.593. Este estudo seguiu os passos recomendados pelo Consolidated criteria for reporting qualitative research (COREQ).

\section{RESULTADOS}

Participaram dez pessoas com estomia, com predominância do sexo feminino (80,0\%), faixa etária de 18 a 92 anos de idade, média de idade de 61,2 anos. $\mathrm{Na}$ caracterização de estado civil houve domínio de viúvos e solteiros, ambos com 40,0\%; e 70,0\% das estomias eram do tipo colostomia resultante de câncer. A maioria dos participantes $(60,0 \%)$ possuía renda de um salário mínimo $(\mathrm{R} \$ 937,00)$, proveniente apenas do auxílio doença de sua nova condição de vida, conforme Tabela 1.

A Fig. 1 reconstitui os itinerários terapêuticos percorridos.

Tabela 1. Caracterização dos participantes do estudo. Crateús (CE), Brasil - 2017.

\begin{tabular}{|c|c|c|c|c|c|c|c|}
\hline $\mathrm{D}$ & Sexo & Idade & $\begin{array}{l}\text { Estado } \\
\text { civil }\end{array}$ & $\begin{array}{l}\text { Tipo de } \\
\text { estomia }\end{array}$ & $\begin{array}{c}\text { Tempo de } \\
\text { convívio (anos) }\end{array}$ & $\begin{array}{l}\text { Etiologia da } \\
\text { estomia }\end{array}$ & $\begin{array}{c}\text { Renda } \\
\text { pessoal (SM) }\end{array}$ \\
\hline D1 & M & 42 & Solteiro & Colostomia & 5 & Síndrome de Fournier & 1 \\
\hline D2 & F & 62 & Solteira & $\begin{array}{c}\text { Colostomia e } \\
\text { urostomia }\end{array}$ & 10 & Câncer de reto & 1 \\
\hline D3 & M & 22 & Casado & Ileostomia & 0,6 & $\begin{array}{c}\text { Perfuração por arma } \\
\text { de fogo }\end{array}$ & 1 \\
\hline D4 & $\mathrm{F}$ & 67 & Casada & Ileostomia & 0,6 & Câncer de Colón & 1 \\
\hline D5 & $\mathrm{F}$ & 92 & Viúva & $\begin{array}{c}\text { Colostomia e } \\
\text { urostomia }\end{array}$ & 5 & Hérnia femoral & 2 \\
\hline D6 & $\mathrm{F}$ & 63 & Solteira & Colostomia & 1 & Câncer colorretal & 1 \\
\hline D7 & $\mathrm{F}$ & 18 & Solteira & Colostomia & 18 & Anomalia anorretal & 1 \\
\hline D8 & F & 76 & Viúva & Colostomia & 0,6 & Câncer de colorretal & 2 \\
\hline D9 & F & 80 & Viúva & Colostomia & 10 & Câncer colorretal & 2 \\
\hline D10 & $\mathrm{F}$ & 90 & Viúva & Colostomia & 4 & $\begin{array}{l}\text { Diverticulite } \\
\text { perfurada }\end{array}$ & 2 \\
\hline
\end{tabular}




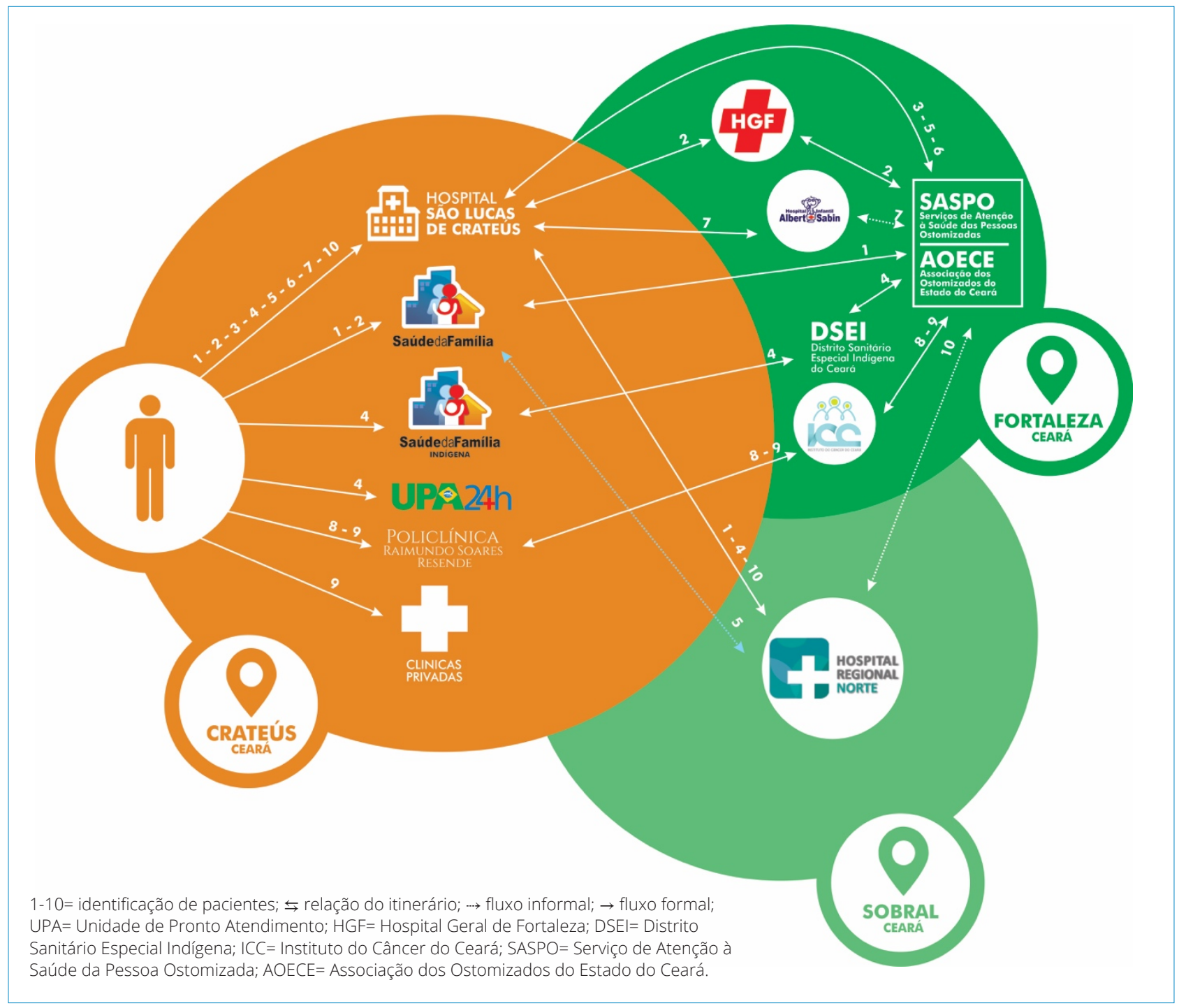

Figura 1. Itinerários terapêuticos de pessoas com estomia. Crateús (CE), Brasil - 2017.

Para melhor compreensão do fluxo, entende-se o traçado contínuo como relação, traçado pontilhado como fluxo informal e seta como fluxo formal. Crateús, cenário do estado com seus serviços de atenção à saúde em distintos níveis de complexidade, município de Sobral (Macrorregião) e Fortaleza, capital do estado, sede do Programa dos Ostomizados e outros serviços de saúde.

Ressalta-se que a Associação dos Ostomizados do Estado do Ceará era responsável até o fim de 2015 pela dispensação de bolsas e atendimento à pessoa com estomia, passando em 2016 esta atenção a ser realizada pela Secretaria da Saúde do Estado do Ceará, com a criação do Serviço de Atenção à Saúde da Pessoa Ostomizada.

Em suma, os caminhos percorridos em busca por atendimento especializado têm início com o surgimento dos sinais e sintomas de doenças, acidentes e/ou agravos em saúde, acessando a priori os serviços de saúde mais próximos e que promovem alívio imediato das suas queixas, como postos de saúde, atendimentos especializados públicos e privados e serviços de atendimento de urgência e emergência. Quando essas não são passíveis de resolução, são melhor investigadas durante o processo de hospitalização (hospital local e hospitais especializados pactuados) e para melhoria do quadro clínico, necessidade de realização de cirúrgica com confecção de estomia.

Movidos pela necessidade continua de cuidados especializados e uso de equipamentos coletores e adjuvantes, esses buscam acesso ao serviço especializado (Serviço de Atenção à Saúde da Pessoa Ostomizada), em sua grande maioria por fluxo formal, sistema de referência/contrareferência. 
A Fig. 2 apresenta a análise de similitude, que gerou leque semântico de palavras mais evocadas nas narrativas, agrupadas em zonas centrais e periféricas, permitindo melhor compreensão do itinerário terapêutico. Destaca-se como termo central "médico", circundado fortemente por vocábulos periféricos "bem", "muito", "fortaleza", "cirurgia"e "sentir". Destarte, os caminhos aqui traçados se estabelecem por meio de sistema da referência do "médico" à "secretaria de saúde" do município (recepção e distribuição dos casos) e, consequentemente, encaminhamento ao serviço especializado - Programa de Atenção à Saúde da Pessoa com Ostomia na capital do estado "Fortaleza".

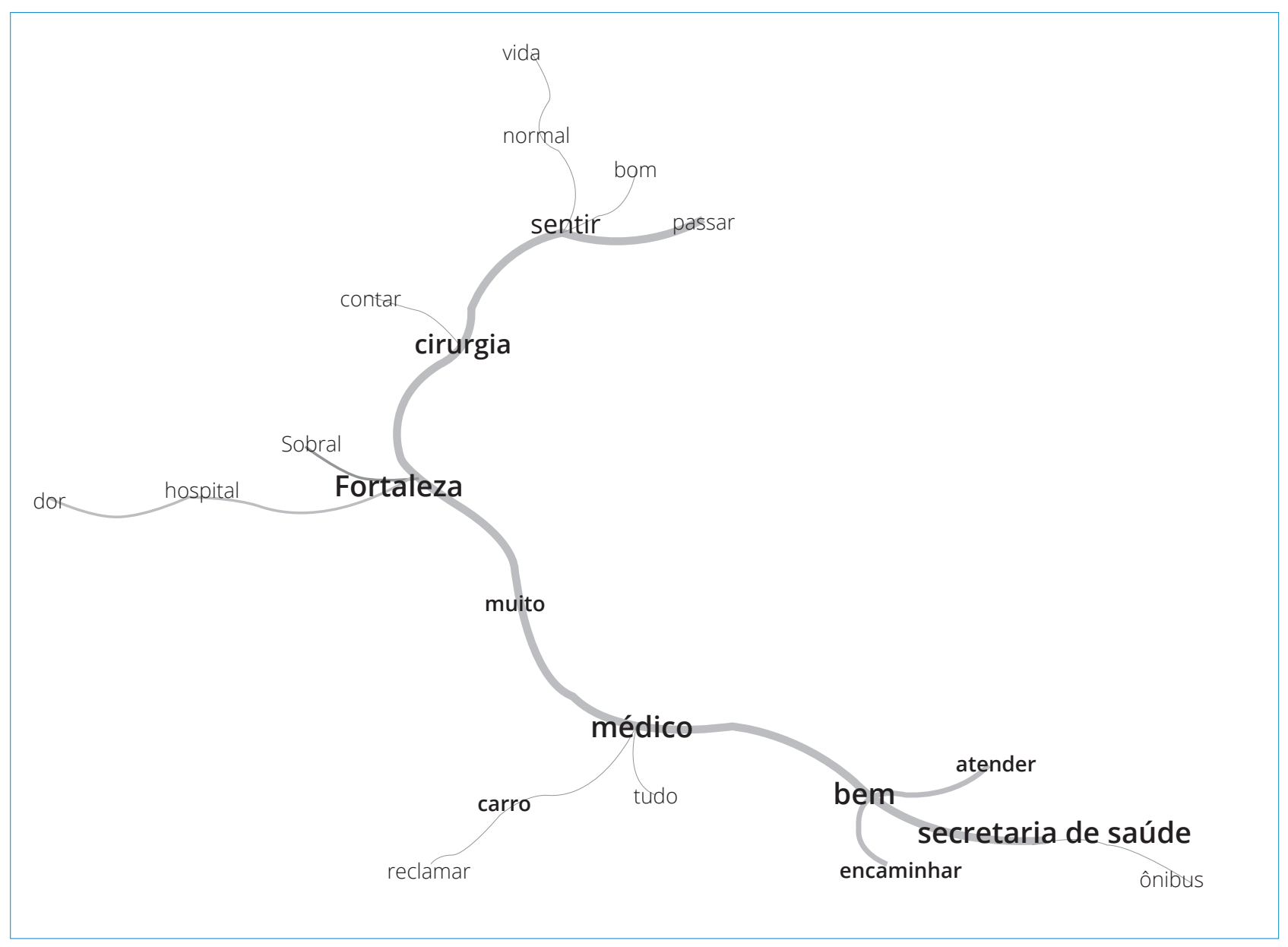

Figura 2. Análise de similitude entre os vocábulos. Crateús (CE), Brasil - 2017.

A sintomatologia é marcada por manifestações gastrointestinais associadas às dores intensas. Entretanto, não é algo linear, processo com momentos distintos, que se inicia com a percepção de que algo diferente está acontecendo no seu corpo, conforme os depoimentos a seguir:

Sentia trovoadas na barriga, muita dor ao evacuar e sangue nas fezes por muito tempo. Procurei o Programa Saúde da Família dos índios, tomei uns remédios, mas não melhorei. Toda vez que ficava com a barriga presa, ia na unidade de pronto atendimento ou no hospital para fazer lavagem. Fiz isso até que o médico do posto estranhou e passou alguns exames. (D4)
No início, sentia muita dor na barriga. Minha barriga começou a crescer, ficar estufada e passava dias e dias sem ir ao banheiro. Tomava chá, tudo o que o povo me ensinava, mas não resolvia, então decidi ir ao posto de saúde. (D6)

Vivia constipada, sentia muita dor, arrotava, coceira e com meu corpo todo vermelho. Paguei uma consulta porque não tinha médico no posto e me pediram para fazer um ultrassom, mas não tinha dinheiro para pagar. Uma amiga conseguiu que eu fizesse na policlínica. (D9)

A narrativa de D1, D3 e D7 é peculiar, pois a gênese da estomia não resulta do câncer colorretal como os demais: 
Fui dormir bem à noite e quando acordei estava com uma bolha que virou uma ferida e, em poucos dias, fui parar no hospital. A ferida aumentou tanto que pegou minhas partes íntimas e ficaram podres (choro). Não gosto nem de lembrar disso. Pensei que fosse morrer. (D1)

Fiquei envolvido com umas 'paradas' e quando tentei sair disso, levei cinco tiros, três na barriga, um no braço e outro na coxa. Tive muita sorte. (D3)

O meu caso é diferente, porque cresci com isso (apontando para a bolsa), então é normal para mim. (D7)

O percurso realizado pelas pessoas com estomia na busca por diagnóstico e tratamento tem início com o aparecimento de sinais e sintomas e busca pelo diagnóstico com consultas e exames. Com a confirmação do diagnóstico, a realização da cirurgia, quimioterapia/radioterapia e os impactos desse tratamento na vida de pessoas com estomia e suas vivências e relações com o serviço especializado, assim os movimentos no itinerário terapêutico se deu pelos sistemas de saúde formais, informais e populares:

Os colegas do PSF. (D1)

O médico. (D2)

Ninguém mim indicou, fui hospitalizado por conta da infecção e o médico achou melhor fazer a cirurgia, para não causar mais infecções. (D3)

Me indicaram de Sobral, primeiro fazia em Sobral, fui para fortaleza, comecei a quimioterapia. (D4)

O médico. (D7)

Fui encaminhada de Sobral para Fortaleza. (D10)

A vizinha me levou ao hospital, e fui encaminhada pelo o médico. (D9)

Secretaria encaminhou, com assistente social. (D6)

O médico. (D5)

Os caminhos e (des)caminhos em busca de serviços especializados pelos depoentes, deram-se das mais variadas formas:

[...] muitas vezes de bicicleta, quando mim desloco pra Sobral ou Fortaleza vou no ônibus. (D1)

Vamos de ônibus. (D2)

O pessoal da secretaria de saúde marcou a consulta [..] para Fortaleza. (D3)
Andei muito! Era do hospital, unidade de pronto atendimento e posto de saúde, até que o hospital daqui (Crateús) me mandou para Sobral de ambulância. Em Sobral, fiz mais uma cirurgia para colocar outra bolsinha. Depois me mandaram ir para Fortaleza fazer o cadastro das bolsas e ficar recebendo por lá. Tudo ajeitado por conta dos índios. (D4)

Pelo ônibus do Gentil Barreiras. (D6)

Através do Gentil Barreiras, nós vamos no carro da prefeitura. (D7)

Fui de ônibus que peguei no Gentil Barreiras. (D5)

Fui de carro próprio. (D8)

Peguei o encaminhamento no Gentil barreiras para Fortaleza. (D9)

Fui encaminhada de Sobral para Fortaleza com os papéis do médico para a associação. Depois fui para o posto (Programa dos Ostomizados). (D10)

Os depoimentos expressaram percursos e movimentos distintos na fase que antecede ao atendimento especializado. Assim como as trajetórias de busca, produção, transporte sanitário e gerenciamento do cuidado para saúde, empreendidos por pessoas, famílias e grupos informais.

\section{DISCUSSÃO}

A partir da análise das falas dos participantes, a busca por uma terapêutica apropriada emerge com a percepção de que algo não está bem, manifestado por alterações de ordens físicas que provocam desconforto. Nesse momento, a pessoa busca formalizar o "diagnóstico", provocando a busca por cuidados e tratamentos. A partir dessa decisão, as escolhas geralmente são aquelas que fazem sentido para cada um, ancoradas em experiências prévias, sempre provisórias. Nesse momento não há um único caminho, cada sujeito faz escolhas dentro das possibilidades que visualiza, são múltiplos e distintos percursos ${ }^{14}$.

A trajetória percorrida tem início com o surgimento dos primeiros sinais e sintomas até o diagnóstico e o encaminhamento para a unidade de tratamento especializado. Alguns acessam de forma direta serviços de urgência e emergência, sejam eles em unidades de pronto atendimento ou hospitais. Algumas vezes sem uma resolução rápida e em outras com diagnóstico e encaminhamento para uma unidade de referência ${ }^{15}$. 
No que se refere ao curso clínico das doenças, principalmente sobre os cânceres, encontra-se imbricado a partir de sinais e sintomas inespecíficos, às vezes, desvalorizados tanto pelo próprio paciente como por profissionais de saúde, em detrimento de barreiras socioculturais ou organizacionais, ocasionando atraso no diagnóstico ${ }^{16}$.

Ressalta-se que a busca, bem como o diagnóstico tardio da doença, demora em iniciar o tratamento e dificuldade de oferecer cuidado e tratamento adequado são fatores determinantes para o agravamento da doença e mortalidade, apontando fragilidade na rede de serviços ${ }^{17}$.

Os serviços de urgência e emergência atuam no atendimento às demandas agudas de forma mais ágil e concentrada, já que a queixa de natureza biológica pode representar um risco à saúde. Outra parcela acessa esses por indisponibilidade de recurso na atenção primária à saúde ou outras dificuldades no acesso aos serviços da atenção primária $^{16}$.

Os diversos níveis de atenção à saúde devem estar capacitados e aptos para atender às necessidades da população e promover cuidado integral. Sua atuação eficiente deve incluir a capacidade de reconhecer os sinais e sintomas, contribuindo, assim, para a suspeição precoce, com o devido encaminhamento ao médico especialista que realizará a confirmação diagnóstica ${ }^{15}$.

Os sistemas de cuidados são construídos por conexões formais, regulares e regulamentadas entre os serviços de saúde, compondo "redes" ou "linhas" de cuidado, em geral desenhadas por gestores e operadas cotidianamente pelos profissionais em suas ações e interações com os usuários, na perspectiva da construção da integralidade do cuidado. Para representar esses trajetos e linhas de cuidado, a imagem de uma "pirâmide" foi a representação historicamente constituída, em especial devido à complexidade crescente dos serviços interligados entre si pelos processos formais de referência/ contrarreferência, com fluxos ascendentes e descendentes que os usuários deveriam seguir ${ }^{18}$.

O “acesso" também pode ser definido como "entrada no sistema”, a obtenção do cuidado julgado necessário (pelos profissionais e/ou pelos usuários), de forma oportuna e qualificada. Ou seja, essa categoria diz respeito a acessar um lugar (um serviço, um profissional, etc.), no tempo adequado (um tempo entre tentar e conseguir) e obter o que precisa (produzir uma resposta, um ato de saúde) ${ }^{19}$. Cabe considerar, contudo, que a definição e qualificação do que significa "lugar adequado", "tempo adequado" e "resposta adequada" depende de quem as enuncia, sendo, portanto, marcadas por dissensos, disputas e conflitos ${ }^{18}$.

O "sistema de saúde"é composto por três subsistemas interrelacionados: o "informal” (familia, comunidade, rede de amigos, grupos de apoio e autoajuda), o "popular" (agentes especializados seculares ou religiosos, mas não reconhecidos legalmente na sociedade) e o subsistema "formal" ou "profissional" (ofertado por agentes governamentais). Os usuários e suas famílias buscam atenção nas três esferas, sem necessariamente seguir um mesmo sentido de percurso ou hierarquia, trilhando itinerários terapêuticos de modos particulares ${ }^{20}$.

Tais caminhos percorridos na busca de soluções para problemas de saúde são, em geral, pouco conhecidos ou relegados a um segundo plano, não sendo um tema prioritário durante a formação profissional em saúde e, também, pouco presentes nas preocupações dos pesquisadores, gestores ou formuladores de políticas. No entanto, podem oferecer uma oportunidade de compreender a complexidade e multiplicidade de movimentos e linhas traçadas no cotidiano pelos usuários, de analisar suas características ou atributos que conferem novos sentidos ao acesso, à utilização e à qualidade dos serviços de saúde na perspectiva dos usuários ${ }^{21}$.

Os depoimentos subsidiaram o desenho de diagramas ou "mapas de cuidado" que foram configurados individualmente e, posteriormente, foram sobrepostos, buscando uma "generalização analítica" para as questões em tela. Os "mapas de cuidado" são formados pelo conjunto de pontos de acesso e cuidado que cada pessoa vai produzindo a partir de suas necessidades e experiências, nos diferentes momentos da vida e nos processos de adoecimento.

Nesse contexto, a atenção primária por ser próxima ao usuário e atender às principais necessidades de saúde, configura-se como ordenador do cuidado. É comum no itinerário constantes peregrinações pela Rede de Atenção à Saúde e pelos serviços de saúde em busca de cuidados e resolução. Fatores socioeconômicos, vulnerabilidade no acesso a estes serviços e comprometimento do vínculo entre famílias e profissionais repercutem na assistência e na busca por atendimento especializado ${ }^{20,22-23}$.

Entretanto, há outra dimensão da constituição de "rede de cuidados à saúde" que escapa da lógica formal dos gestores do sistema sanitário e que configura outro modo do usuário acessar, utilizar e atribuir significados de qualidade aos serviços de saúde a que recorre nas situações de adoecimento. Esses constituem os movimentos reais dos usuários e os modos como eles constroem seus "itinerários terapêuticos 
singulares" no sistema de saúde, questão compreendida no âmbito desta pesquisa ${ }^{3}$.

Sabe-se que a organização de uma rede de serviços de saúde deve primar por fluxo centrado nas necessidades peculiares de determinado grupo social. Baseado nesse prisma e considerando a pessoa com estomia como ser único e singular, torna-se imprescindível elaborar políticas públicas de saúde que busquem continuamente aperfeiçoar e (re)organizar as demandas de cuidado para a promoção da saúde ${ }^{22}$.

Destaca-se como fator limitante para esse estudo o número reduzido de participantes. Tal fato não possibilitou aplicação de outras alternativas de análise pelo IRaMuTeQ em detrimento ao tamanho do corpus textual.

\section{CONCLUSÃO}

O caminho percorrido pelas pessoas com estomia ao serviço especializado se dá com o surgimento dos sinais e sintomas, busca pelo diagnóstico, tratamento (atenção primária, serviços especializados e ambulatórios) e, consequentemente, intervenção cirúrgica (hospitais), sendo o acesso marcado por conexões formais, sustentados por subsistemas inter-relacionados: o "informal", o "popular" e o subsistema "formal".

Compreender como se produzem as linhas de cuidados à pessoa com estomia proporciona a identificação de necessidades básicas apresentadas por essa clientela, (re) conhecendo as facilidades e barreiras encontradas no percurso empreendido até que se obtenha um diagnóstico preciso e que se implemente o plano terapêutico adequado/especializado e as relações sociais cotidianas nesse interstício.

\section{CONTRIBUIÇÃO DOS AUTORES}

Conceitualização: Marques ADB; Metodologia: Marques ADB, Florêncio RS e Cestari VRF; Investigação: Silva EA, Vidal SAS e Marques ADB; Redação - Primeira versão: Marques ADB; Recursos: Marques ADB, Silva EA e Vidal SAS; Supervisão: Moreira TMM.

\section{REFERÊNCIAS}

1. Silveira CB, Costa LSP, Jorge MSB. Redes de atenção à saúde como produtoras de cuidado em saúde mental: uma análise reflexiva. Rev Port Enferm Saúde Mental 2018 jun;19:61-70. https://doi.org/10.19131/rpesm.0203

2. Santos L. Região de saúde e suas redes de atenção: modelo organizativo-sistêmico do SUS. Cien Saude Colet 2017 abr;22(4):1281-9. https://doi.org/10.1590/141381232017224.26392016

3. Silva NEK, Sancho LG, Figueiredo WS. Entre fluxos e projetos terapêuticos: revisitando as noções de linha do cuidado em saúde e itinerários terapêuticos. Cien Saude Colet 2016 mar; 21(3):843-52. https://doi.org/10.1590/141381232015213.08572015

4. Carnut L. Cuidado, integralidade e atenção primária: articulação essencial para refletir sobre o setor saúde no Brasil. Saúde Debate 2017 dez;41(115):1177-86. https://doi. org/10.1590/0103-1104201711515

5. Portaria n. 4.279 de 30 de dezembro de 2010 (BR). Estabelece diretrizes para a organização da Rede de Atenção à Saúde no âmbito do Sistema Único de Saúde (SUS). Diário Oficial da União [periódico na internet], Brasília (DF). 30 dez 2010 [citado 13 mai 2019]. Disponível em: https://conselho.saude.gov.br/ultimas_noticias/2011/ img/07_jan_portaria4279_301210.pdf
6. Portaria n. 793, de 24 de abril de 2012 (BR). Institui a rede de cuidados à pessoa com deficiência no âmbito do Sistema Único de Saúde. Diário Oficial da União [periódico na internet], Brasília (DF). 24 abr 2012. [citado 13 mai 2019]. Disponível em: https://bvsms.saude.gov.br/bvs/saudelegis/ gm/2012/prt0793_24_04_2012.html

7. Portaria n. 400, de 16 de novembro de 2009 (BR). Considerando a política nacional de saúde da pessoa com deficiência, instituída pela Portaria n. 1.060/GM, de 05 de junho de 2002. Diário Oficial da União [periódico na internet], Brasília (DF). 18 nov 2009 [citado 13 mai 2019]. Disponível em: http://bvsms.saude.gov.br/bvs/saudelegis/ sas/2009/prt0400_16_11_2009.html

8. Resolução normativa - RN n. 325 de 18 de abril de 2013 (BR). Altera a Resolução Normativa - RN n. 211, de 11 de janeiro de 2010, que dispõe sobre o Rol de Procedimentos e Eventos em Saúde no âmbito da Saúde Suplementar, para regulamentar o fornecimento de bolsas de colostomia, ileostomia e urostomia, sonda vesical de demora e coletor de urina com conector, de que trata art. 10-B da Lei n 9.656, de 1998. Diário Oficial da União [periódico na internet], Brasília (DF). 18 abr 2013 [citado 13 mai 2019]. Disponível em: https://bvsms.saude.gov.br/bvs/saudelegis/ans/2013/ res0325_18_04_2013.html 
9. Moraes JT. Serviços de atenção ao estomizado: análise diagnóstica no Estado de Minas Gerais, Brasil. Cad Saúde Colet 2014 jan/mar;22(1):101-8. https://doi. org/10.1590/1414-462X201400010015

10. Secretaria da Saúde do Estado do Ceará. Programa comemora Dia dos Ostomizados com crescimento de 63\% [Internet]. 2017 [citado 2019 Maio 13]. Disponível em: https:// www.ceara.gov.br/2017/11/16/programa-comemora-diados-ostomizados-com-crescimento-de-63/

11. Ministério da Saúde (BR). Agenda nacional de prioridades de pesquisa em saúde. $2^{\mathrm{a}}$ ed. Brasília (DF): Ministério da Saúde; 2015.

12. Minayo MCS. Amostragem e saturação em pesquisa qualitativa: consensos e controvérsias. Rev Pesqui Qual [Internet]. 2017 abr; [citado 2019 mai 13]; 5(7):1-12. Disponível em: https://editora.sepq.org.br/index.php/rpq/ article/view/82

13. Souza MAR, Wall ML, Thuler ACMC, Lowen IMV, Peres AM. O uso do software IRAMUTEQ na análise de dados em pesquisas qualitativas. Rev Esc Enferm USP 2018 out; 52:e03353. https://doi.org/10.1590/s1980-220×2017015003353

14. Brustolin A, Ferretti F. Itinerário terapêutico de idosos sobreviventes ao câncer. Acta Paul Enferm 2017 jan/ fev;30(1):47-59. https://doi.org/10.1590/1982-0194201700008

15. Lima BC, Silva LF, Góes FGB, Ribeiro MTS, Alves LL. O itinerário terapêutico de famílias de crianças com câncer: dificuldades encontradas neste percurso. Rev. Gaúcha Enferm 2018 out;39:e20180004. https://doi.org/10.1590/19831447.2018.20180004

16. Souza KA, Souza SR, Tocantins FR, Freitas TF, Pacheco PQC. O itinerário terapêutico do paciente em tratamento oncológico: implicações para a prática de enfermagem.
Cienc Cuid Saude 2016 abr/jun;15(2):259-67. https://doi. org/10.4025/cienccuidsaude.v15i2.29896

17. Foletto EF, Jackisch SE, Dotto ML, Severo C, Pappen E, Valim ARM et al. Therapeutic itinerary of colorectal cancer patients treated in the state of Rio Grande do Sul. J Coloproctol 2016 abr/jun;36(2):91-6. https://doi.org/10.1016/j.jcol.2016.03.008

18. Lazarino MSA, Silva TL, Dias EC. Apoio matricial como estratégia para o fortalecimento da saúde do trabalhador na atenção básica. Rev bras saúde ocup 2019 jun;44:e23. https://doi.org/10.1590/2317-6369000009318

19. Souza FF, Zambenedetti G. Percursos de cuidado: pistas sobre itinerários terapêuticos em saúde mental. Tempus Actas Saúde Colet 2018 ago;11(4):105-22. https://doi. org/10.18569/tempus.v11i4.2485

20. Siqueira SMC, Jesus VS, Camargo CL. Itinerário terapêutico em situações de urgência e emergência pediátrica em uma comunidade quilombola. Ciênc Saúde Coletiva 2016 jan; 21(1):179-89. https://doi.org/10.1590/141381232015211.20472014

21. Silva JG, Branco JGO, Vieira LJES, Brilhante AVM, Silva RM. Direitos sexuais e reprodutivos de mulheres em situação de violência sexual: o que dizem gestores, profissionais e usuárias dos serviços de referência? Saude soc 2019 abr/jun;28(2):187200. https://doi.org/10.1590/s0104-12902019180309

22. Luz RO, Pieszak GM, Arrué AM, Gomes GC, Neves ET, Rodrigues AP. Itinerário terapêutico de famílias de crianças com necessidades especiais de saúde. Rev Rene 2019; 20:e33937. https://doi.org/10.15253/21756783.20192033937

23. Figueiredo PA, Alvim NAT. Diretrizes para um programa de atenção integral a pacientes estomizados e familiares: uma proposta de enfermagem. Rev Latino-Am Enfermagem 2016; 24:e2694. https://doi.org/10.1590/1518-8345.0507.2694 